\title{
UTILIZAÇÃO DO SISTEMA PIEZOELÉTRICO EM CIRURGIAS BUCAIS: INDICAÇÕES, VANTAGENS E DESVANTAGENS
}

\author{
PIEZOELETRICT SYSTEM IN ORAL SURGERY: \\ INDICATIONS, ADVANTAGES AND DISADVANTAGES
}

Marcos Antônio Lima de Carvalho', Gabriel Marques ${ }^{2}$, Guilherme dos Santos Trento ${ }^{3}$, Luis Eduardo Marques Padovan ${ }^{4}$, Leandro Eduardo Klüppel ${ }^{5}$

\begin{abstract}
Autor para correspondência: Guilherme dos Santos Trento - guilhermetrento@yahoo.com.br 'Mestre em Implantodontia. Dentista na Implant Health, Manaus, Amazonas, Brasil.

${ }^{2}$ Mestre em Implantodontia. Dentista na Prefeitura Municipal de Itajaí, Santa Catarina, Brasil.

${ }^{3}$ Doutorado em andamento em Ciências Odontológicas na Universidade Estadual Paulista Júlio de Mesquita Filho - UNESP, São Paulo, Brasil.

${ }^{4}$ Doutor em Odontologia. Professor no Instituto Latino Americano de Pesquisa e Ensino Odontológica - ILAPEO, Curitiba, Paraná, Brasil. ${ }^{5}$ Doutor em Cirurgia e Traumatologia Buco-Maxilo-Facial. Dentista no Hospital XV, Serviço de Cirurgia Buco-Maxilo-Facial, Curitiba, Paraná, Brasil.
\end{abstract}

RESUMO I A ostectomia é uma manobra cirúrgica fundamental que pode afetar a reparação tecidual de modo a aumentar ou reduzir a morbidade do procedimento. O tipo de equipamento e/ ou instrumental utilizado para a ostectomia pode influenciar diretamente no reparo ósseo. $O$ objetivo deste trabalho foi realizar uma revisão bibliográfica a respeito da utilização do sistema piezoelétrico em cirurgias orais e maxilofaciais, avaliando as suas indicações, vantagens e desvantagens. Para a elaboração desta revisão crítica da literatura foi realizada uma busca de artigos científicos no PUBMED até $\circ$ ano de 2012 . Segundo os critérios de inclusão e exclusão propostos pelos autores, foi encontrado um total de 28 artigos compatíveis com o objetivo do trabalho. Como conclusão, pode-se afirmar que o sistema piezelétrico tem demonstrado ser uma técnica cirúrgica muito promissora indicada para cirurgias orais, proporcionando osteotomias precisas, limpas e com mínimo de trauma para os tecidos moles. No entanto, há o desgaste acentuado das pontas ultrassônicas elevando o custo da utilização do sistema e aumentando o tempo em alguns procedimentos.

\begin{abstract}
Ostectomy is a surgical maneuver that can affect tissue repair, increasing the overall morbidity of the procedure. The type of equipment or surgical armamentarium can directly influence bone repair. The aim of this study was to perform a literature review concerning the use of piezoelectric technology in oral and maxillofacial surgical procedures evaluating its indications, advantages and disadvantages. For the preparation of this critical review of the literature, a search of scientific articles was carried out in PUBMED until the year 2012. According to the inclusion and exclusion criteria porposed by the authors, a total of 28 articles were found compatible to the objective of the study. As a conclusion, it can be stated that the piezoeletric system has been shown to be a very promising surgical technique indicated to oral surgeries, and providing accurate, clean, and minimally traumatic osteotomies for the soft tissues. However, there is na accentuated wear of the ultrasonic tips increasing the costs of the system and the time spent in the procedures.
\end{abstract}

Keywords: Piezosurgery; surgery; dental implants

Palavras-chave: Piezocirurgia; Cirurgia; Implantes dentários. 


\section{INTRODUÇÃO}

A utilização de instrumentos ultrassônicos na odontologia vem sendo descrita desde a metade do século passado. Entretanto, na área da cirurgia oral, a utilização destes dispositivos foi descrita na década de 1980 com o uso clínico do ultra-som em cirurgias ósseas'. Contudo, apenas no final da década de 1990 começaram a surgir trabalhos com a utilização de instrumentos ultrassônicos como substitutos aos instrumentos rotatórios na cirurgia oral e maxilofacial - um dispositivo ultrassônico baseado no efeito piezoelétrico reverso -, iniciandose um novo conceito chamado de Piezocirurgia. $O$ objetivo é de proporcionar cirurgias ósseas com maior segurança para os tecidos moles e precisão nas osteotomias além de um excelente reparo tecidual $\left.\right|^{2-4}$.

O interesse pela piezocirurgia tem aumentado nos últimos anos, principalmente pelas vantagens propostas pelos fabricantes dos equipamentos e, conseqüentemente, $\bigcirc$ fato haver estudos demonstrando as suas utilizações nas mais diversas áreas: cirurgias otorrinolaringológicas, na correção de defeitos estéticos ou funcionais ${ }^{5-6}$, na cirurgia de cabeça e pescoço para a remoção de tumores ${ }^{7-8}$, na cirurgia crânio e bucomaxilofacial ${ }^{9-13}$.

Dentro da cirurgia oral e maxilofacial, o dispositivo piezoelétrico tem sido utilizado na remoção de elementos dentais inclusos e ou impactados, exérese de lesões em tecido ósseo próximas a estruturas nobres ${ }^{14-15}$. Outra forma de utilização consiste na confecção de osteotomias para a realização de distração osteogênica com a finalidade de tratamento das deformidades maxilomandibulares e alvéolodentárias, nas quais o dispositivo tem demonstrado bons resultados quanto à simplificação da técnica cirúrgica e reduzindo a incidência de complicações transoperatórias ${ }^{13,16}$. Na área da Implantodontia, esta técnica pode ser indicada para procedimentos para levantamento do seio maxilar, remoção de enxerto ósseo ou mesmo para confecção do leito onde será instalado o implante osteointegrado.

O dispositivo piezoelétrico consiste em um equipamento composto por uma peça de mão, com vários tipos de pontas de trabalho, a qual é conectada a uma fonte eletrônica central que converte a corrente elétrica em ondas que emitem vibrações ultrassônicas a uma freqüência de 25 e $30 \mathrm{kHz}$, com oscilação (amplitude) de 60 a $210 \mu \mathrm{m}$ e potência de até $50 \mathrm{~W}$. $O$ equipamento ainda possui uma bomba peristáltica responsável pelo fluxo do liquido de resfriamento, o que permitiria um corte seletivo apenas em estruturas mineralizadas sem danificar tecidos moles ${ }^{5,717}$. Por fim, este trabalho tem como objetivo apresentar uma revisão da literatura sobre indicações, vantagens e desvantagens desta técnica.

\section{MATERIAL E MÉTODOS}

Este trabalho científico é uma revisão crítica da literatura. Os artigos foram selecionados pelos autores na base de dados em literatura médica e odontológica internacional MEDLINE.

Como estratégia de busca, foram avaliados artigos publicados na língua inglesa até o mês de outubro do ano de 2012, empregando os termos listados abaixo:

1) Piezoelectric surgery (or ultrasonic system) and bone surgery

2) Piezoelectric surgery (or ultrasonic system) and dental implants

3) Piezoelectric surgery (or ultrasonic system) and bone grafts

4) Piezoelectric surgery (or ultrasonic system) and bone healing

5) Piezoelectric surgery (or ultrasonic system) and maxillofacial

\section{Seleção dos artigos}

Foram incluídos artigos científicos que apresentavam 
séries de casos, bem como estudos randomizados envolvendo animais e seres humanos. Eventualmente, artigos científicos do tipo nota técnica foram incluídos quando os autores julgaram pertinente.

A seleção dos estudos procedeu-se de acordo com a sequencia a seguir:

1) Avaliação dos títulos dos artigos identificados através da aplicação das estratégias de busca;

2) Avaliação dos resumos;

3) Leitura integral dos artigos pertinentes ao assunto.

Após completada a parte das buscas dos artigos, foram encontrados um total de 53 artigos que se encaixavam conforme os critérios de seleção. Neste momento, 25 artigos foram excluídos das pesquisa por não serem compatíveis com os critérios propostos, resultando num total de 28 artigos.

\section{REVISÃO DA LITERATURA}

No ano de 1880, os irmãos Curie observaram que certos materiais (quartzo, turmalina, topázio), quando submetidos a estresse mecânico, produziam cargas elétricas em sua superfície - a este fenômeno deram $\circ$ nome de piezoeletricidade. Anos mais tarde, Gabriel Lippmann descobriu um efeito inverso, onde observa que, quando induzido um campo elétrico nestes materiais, estes reagem produzindo deformações mecânicas - efeito este denominado piezoelétrico inverso/reverso ${ }^{18}$.

Desde então o efeito piezoelétrico vem sendo aplicado nas mais diversas áreas técnicas, tais como: comunicação e controles (rádios, celulares, televisões, radares automotivos), indústria (transdutores, sensores, bombas, motores, lavadores ultrassônicos), área médica (os equipamentos ultrassônicos emitem ondas mecânicas inaudíveis e biologicamente inofensivas). Esse simples fenômeno de agitação induz uma desorganização e fragmentação de todas as interfaces entre corpos de natureza diferente. As vibrações ultrassônicas permitem a clivagem de interfaces sólido-sólido (por diferencial de vibração) e de interfaces sólido-líquido (por cavitação) ${ }^{19-20}$.

A utilização deste efeito dentro da área médica acontece em virtude da descoberta de que este possibilitaria, nas cirurgias onde se faz necessária a realização de osteotomias e/ou estectomias, um corte seletivo do tecido ósseo (atuando apenas no tecido ósseo, preservando tecidos moles). Tal instrumento vêm ganhando espaço frente ao uso de técnicas convencionais e instrumentos rotatórios e oscilatórios. Dentro da cirurgia maxilofacial, as osteotomias são corriqueiras. Em virtude disto, a piezocirurgia tem sido indicada para a execução de diversos procedimentos, onde podemos destacar: a cirurgia ortognática com as osteotomias Le Fort I, associada ou não a expansões palatais e osteotomias sagitais dos ramos, osteotomias Le Fort III para o tratamento de pacientes sindrômicos, utilização na remoção de enxerto ósseo, osteotomias para o tratamento de patologias $^{9-11}$.

Em cirurgias orais, as vantagens se aplicam à proteção de estruturas nobres, como o nervo alveolar inferior (NAl), e na reduzindo do edema pós-operatório, pelo menor trauma cirúrgico ${ }^{12,14,15}$. Em cirurgias de grande porte, 0 dispositivo tem demonstrado bons resultados quanto à simplificação da técnica cirúrgica, reduzindo a incidência de complicações transoperatórias ${ }^{13,16}$.

Na implantodontia, muitas vezes se faz necessário a realização de procedimentos que visam recompor - volume ósseo, suficiente para a instalação dos implantes. Sendo assim, diversas técnicas de enxertia óssea, com o uso de osso autógeno em bloco ou particulado para a reconstrução desses defeitos podem ser executadas com o uso do sistema piezoelétrico.

Além das técnicas de enxertia óssea, técnicas de expansões ósseas com $\circ$ intuito de aumentar a espessura óssea e, simultaneamente, instalação de implantes, vem sendo utilizadas com sucesso obtendo expansões imediatas de até 9 milímetros ${ }^{17-25}$.

Ainda, a técnica de "sinus lifting" tem sido utilizada com excelentes resultados desde os anos 1980 e, desde então, já sofreu diversas modificações. Atualmente consiste em um acesso ao seio maxilar 
pela parede lateral do seio maxilar. Um fator importante é manter a membrana de Schneider intacta para que, uma vez levantada, torne-se o sítio receptor para o enxerto ósseo, estabilizando o material de enxerto durante o período de reparo. A principal complicação na utilização desta técnica consiste na possibilidade de perfuração acidental da membrana. A taxa de perfuração relatada pela literatura varia de 14 a $56 \%$ com a utilização de instrumentos manuais e rotatórios. A utilização da piezocirurgia para a elevação do seio maxilar tem aumentado, em virtude da proposta deste aparelho de apresentar um corte seletivo para tecidos duros, preservando os tecidos moles de possíveis lesões causadas por instrumentos rotatórios.

Conseqüentemente, diminui o risco de perfuração da membrana durante os procedimentos e aumenta a visibilidade trans-operatória devido a um melhor controle no sangramento, além de proporcionar um maior conforto operatório ${ }^{26-28}$.

\section{DISCUSSÃO}

A utilização da piezocirurgia vem sendo indicada nos mais diversos procedimentos dentro da cirurgia oral e maxilofacial devido a uma série de vantagens proposta pelos fabricantes $3-7,10,12-14,16,21-24$ e, por ser ainda uma técnica relativamente nova, diversos questionamentos vêm sendo feito sobre as vantagens quando comparadas às técnicas convencionais que utilizam instrumentos rotatórios e reciprocantes.

A utilização do dispositivo piezoelétrico tem sido associada a uma característica chamada de "corte seletivo", no qual a vibração ultrasônica das ponteiras atuariam de forma específica em tecidos mineralizados através do efeito de cavitação, causando a ruptura desta estrutura e, consequentemente, promovendo o corte deste ${ }^{20}$.

Dentre as principais vantagens inerentes a técnica, a literatura tem confirmado a proposta dos fabricantes quanto a característica do equipamento em realizar cortes ósseos de forma precisa, proporcionando uma melhor visualização do campo operatório, com ausência de injurias à tecidos moles (dura- máter, mucosas e tecido nervoso), além de uma diminuição do sangramento trans-operatório, com menor vibração, reduzindo desta forma o estresse do paciente em procedimentos sob anestesia local, quando comparados aos instrumentos convencionais", $11,13,16,21,22,26-28$.

No que se refere ao trauma gerado e processo de reparo do tecido ósseo, um estudo em modelo animal demonstrou que o uso da piezocirurgia proporciona melhor reparo tecidual que os instrumentos rotatórios ${ }^{4}$. Além disso alguns autores comprovaram a viabilidade da utilização do osso coletado com o uso do dispositivo ${ }^{23,24}$.

Outra vantagem relacionada ao uso da piezocirurgia está na realização de procedimentos próximo a estruturas nobres, como vasos e nervos, demonstrando excelentes resultados, minimizando e, em alguns casos, evitando lesões, mesmo quando ocorre o contato direto do dispositivo com estas estruturas $^{14-15}$. Quando utilizado em procedimentos de elevação do seio maxilar, onde se faz necessária a preservação da membrana de Schneider, melhores resultados tem se observado, principalmente pela diminuição do risco de perfuração desta ${ }^{26-28}$, além de proporcionar uma redução do edema pósoperatório pelo menor trauma cirúrgico ${ }^{12}$.

Entretanto, o fato do dispositivo piezoelétrico apresentar grande precisão de corte, a sua eficiência implica em um desgaste prematuro das pontas causando uma diminuição na velocidade de corte. Alguns autores sugerem utilizar 10 vezes cada ponta ultrasônica, no máximo, pois apesar do revestimento de nitreto de titânio ou diamante, as ponteiras desgastam rapidamente causando danos aos tecidos pelo aquecimento. Ainda, consideram, por vezes, o dispositivo ultrasônico menos eficiente quando comparado a instrumentos tradicionais (instrumentos rotatórios de alto poder de corte), principalmente quando submetido a procedimentos em estrutura óssea altamente densa, levando o profissional experiente muitas vezes a retardar alguns procedimentos que seriam normalmente mais rápidos utilizando a técnica convencional ${ }^{20}$. 


\section{CONCLUSÃO}

Podemos concluir após a revisão da literatura que o sistema piezelétrico tem se demonstrado com uma técnica cirúrgica muito promissora, podendo ser indicada nas mais diversas áreas da cirurgia bucal. Este sistema proporciona osteotomias precisas, limpas e com mínimo de trauma para os tecidos moles, além de diminuir o sangramento transoperatório, quando comparado a cirurgia convencional com uso de instrumentos rotatórios, porém essa precisão está intimamente ligada ao desgaste acentuado das pontas ultrassônicas elevando o custo da utilização do sistema e aumentando o tempo em alguns procedimentos.

\section{CONTRIBUIÇÕES DOS AUTORES}

Carvalho MAL participou da concepção, delineamento, busca e análise descritiva dos dados da pesquisa, interpretação dos resultados, redação do artigo científico. Marques $G$ participou da coleta dos dados da pesquisa, interpretação dos resultados, redação do artigo científico. Trento GS participou da coleta dos dados da pesquisa, interpretação dos resultados, redação do artigo científico e encaminhamento do artigo científico. Padovan LEM participou da concepção, delineamento, análise estatística dos dados da pesquisa, interpretação dos resultados, redação. Klüppel LE participou da concepção, delineamento, análise estatística dos dados da pesquisa, interpretação dos resultados, redação.

\section{CONFLITOS DE INTERESSES}

Nenhum conflito financeiro, legal ou político envolvendo terceiros (governo, empresas e fundações privadas, etc.) foi declarado para nenhum aspecto do trabalho submetido (incluindo mas não limitandose a subvenções e financiamentos, conselho consultivo, desenho de estudo, preparação de manuscrito, análise estatística, etc).

\section{REFERÊNCIAS}

1. Horton JE, Tarpley TM Jr, Jacoway JR. Clinical applications of ultrasonic instrumentation in the surgical removal of bone. Oral Surg Oral Med Oral Pathol. $1981 ; 51$ (3):236-42

2. Torrella F, Pitarch J, Cabanes G, Anitua E. Ultrasonic ostectomy for the surgical approach of the maxillary sinus: a technical note. Int J Oral Maxilllofac Implants. 1998; 13(5):697-700

3. Vercellotti T, De Paoli S, Nevins M. The piezoelectric bony window osteotomy and sinus membrane elevation: introduction of a new technique for simplification of the sinus augmentation procedure. Int J Periodontics Restorative Dent. $2001 ; 21(6): 561-7$

4. Vercellotti T, Nevins ML, Kim DM, Nevins M, Wada K, Schenk RK et al. Osseous response following resective therapy with piezosurgery. Int J Periodontics Restorative Dent. $2005 ; 25(6): 543-49$

5. Salami A, Dellepiane M, Proto E, Mora R. Piezosurgery in otologic surgery: four years of experience. Otolaryngol Head Neck Surg. 2009; 140(3):41 2-18. doi: 10.1016/i. otohns.2008.11.013

6. Robiony M, Polini F, Costa F, Toro C, Politi M. Ultrasound piezoelectric vibrations to perform osteotomies in rhinoplasty. J Oral Maxillofac Surg. 2007;65(5):1035-8. doi: 10.1016/i. joms.2005.11.082

7. Salami A, Dellepiane M, Crippa B, Mora R. A new method for osteotomies in oncologic nasal surgery: Piezosurgery. Am J Otolaryngol. 2010;31(3):150-3. doi: 10.1016/i. amioto.2008.12.001

8. Gleizal A, Béra JC, Lavandier B, Béziat JL. Voies d'abord craniofaciales des tumeurs orbitaires et découpe osseuse ultrasonique. J Fr Ophtalmol. 2007;30(9):882-91

9. Beziat JL, Bera JC, Lavandier B, Gleizal A. Ultrasonic osteotomy as a new technique in craniomaxillofacial surgery. Int J Oral Maxillofac Surg. 2007;36(6):493-500. doi: 10.1016/i.ijom.2007.01.012

10. Robiony M, Polini F, Costa F, Sembronio S, Zerman $N$, Politi M. Endoscopically assisted intraoral vertical ramus osteotomy and piezoelectric surgery in mandibular prognathism. J Oral Maxillofac Surg. 2007;65(10):21 19-24. doi: $10.1016 /$ i.joms.2006.03.048

11. Landes CA, Stübinger S, Rieger J, Williger B, Ha TK, Sader R. Critical evaluation of piezoelectric osteotomy in orthognathic surgery: operative technique, blood loss, time requirement, nerve and vessel integrity. J Oral Maxillofac Surg. 2008;66(4):657-74. doi: 10.1016/i.joms.2007.06.633

12. Sortino F, Pedullà E, Masoli V. The piezoelectric and rotatory osteotomy technique in impacted third molar surgery: comparison of postoperative recovery. J Oral Maxillofac Surg. 2008;66(1 2):2444-8. doi: 10.1016/i. joms.2008.06.004

13. Heiland M, Blessmann M, Pohlenz P, Li L, Schmelzle $\mathrm{R}$, Blake F. Intraoral osteotomies using piezosurgery for distraction in an infant with Pierre-Robin sequence. Clin Oral Investing. 2007;1 1(3):303-6. doi: 10.1007/s00784-006$0091-y$

14. Degerliyurt K, Akar V, Denizci S, Yucel E. Bone lid technique with piezosurgery to preserve inferior alveolar nerve. Oral Surg Oral Med Oral Pathol Oral Radiol Endod. 
15. Schaeren $S$, Jaquiéry $C$, Heberer $M$, Tolnay $M$, Vercellotti $T$, Martin I. Assessment of nerve damage using a novel ultrasonic device for bone cutting. J Oral Maxillofac Surg. 2008;66(3):593-6. doi: 10.1016/i.joms.2007.03.025

16. González-García A, Diniz-Freitas $M$, Somoza-Martín $M$, García-García A. Piezoelectric and conventional osteotomy in alveolar distraction osteogenesis in a series of 17 patients. Int J Oral Maxillofacial Implants. 2008;23(5):891-6

17. Kfouri FA, Duailibi MT, Bretos JLG, Ferreira LM, Duailibi SE. Cirurgia piezoelétrica em implantodontia: aplicações clínicas. Rev Gaucha Odontolol. 2009;57(1);121-6

18. Ballato A. Piezoelectricity: old effect, new thrusts. IEEE transactions on ultrasonics, ferroeletrics, and frequency control 1995; 12(5):916-26. doi: $10.1109 / 58.464826$

19. Ballato A. Piezoeletricity: history and new thrusts. IEEE ultrasonics symposium 1996; 1:575-83. doi: $10.1109 /$ ULTSYM.1996.584046

20. Leclercq P, Zenati C, Amr S, Dohan DM. Ultrasonic bone cut part 1: state-of-the-art technologies and common applications. J Oral Maxillofac Surg. 2008;66(1):177-82. doi: 10.1016/i.joms.2005.12.054

21. Sohn DS, Ahn MR, Lee WH, Yeo DS, Lim SY. Piezoelectric osteotomy for intraoral harvesting of bone blocks. Int J Periodontics Restorative Dent. 2007;27(2):1 27-31

22. Happe A. Use of a piezoelectric surgical device to harvest bone grafts from the mandibular ramus: report of 40 cases. Int J Periodontics Restorative Dent. 2007;27(3):241-9

23. Sivolella S, Berengo M, Scarin M, Mella F, Martinelli F. Autogenous particulate bone collected with a piezo-electric surgical device and bone trap: a microbiological and histomorphometric study. Arch Oral Biol. 2006;51(10):88391. doi: $10.1016 /$ i.archoralbio.2006.04.001

24. Chiriac G, Herten M, Schwarz F, Rothamel D, Becker J. Autogenous bone chips: influence of a new piezoelectric device (Piezosurgery) on chip morphology, cellviability and differentiation. J Clin Periodontol. 2005;32(9):994-9. doi: $10.1111 /$ i.1600-051X.2005.00809.x

25. Blus C, Szmukler-Moncler S. Split-crest and immediate implant placement with ultra-sonic bone surgery: a 3-year life-table analysis with 230 treated sites. Clin Oral Implants Res. 2006;17(6):700-7. doi: 10.1111/i.16000501.2006.01206.x

26. Wallace SS, Mazor Z, Froum SJ, Cho SC, Tarnow DP. Schneiderian membrane perforation rate during sinus elevation using piezosurgery: clinical results of 100 consecutive cases. Int J Periodontics Restorative Dent. 2007;27(5):413-9
27. Blus C, Szmukler-Moncler S, Salama M, Salama H, Garber $D$. Sinus bone grafting procedures using ultrasonic bone surgery: 5-year experience. Int J Periodontics Restorative Dent. 2008;28(3):221-9

28. Barone A, Santini S, Marconcini S, Giacomelli L, Gherlone $\mathrm{E}$, Covani U. Osteotomy and membrane elevation during the maxillary sinus augmentation procedure. a comparative study: piezoelectric device vs. conventional rotative instruments. Clin Oral Implants Res. 2008;19(5):51 1-15. doi: $10.1111 /$ i.1600-0501.2007.01498.x 